
\title{
$\begin{array}{ll}\text { Research Square } & \text { Preprints are preliminary reports that have not undergone peer review. } \\ \text { They should not be considered conclusive, used to inform clinical practice, } \\ \text { or referenced by the media as validated information. }\end{array}$
}

\section{SIX1 Down-Regulation Influence Drug Resistance, Epithelial-Mesenchymal Transcription Factors and Self-Renewal Capacity in Hepatocellular Carcinoma}

Pelin Balcik Ercin ( $\square$ pbalcik@gtu.edu.tr)

Gebze Technical University https://orcid.org/0000-0002-3470-0393

\section{Arzu Aysan}

Gebze Technical University: Gebze Teknik Universitesi

Nazli Salik

Gebze Technical University: Gebze Teknik Universitesi

\section{Esma Erciyas}

Gebze Technical University: Gebze Teknik Universitesi

\section{Research Article}

Keywords: SIX1, Epithelial-Mesenchymal Transition, HCC, Drug Response, CD90

Posted Date: April 29th, 2021

DOI: https://doi.org/10.21203/rs.3.rs-376584/v1

License: (9) This work is licensed under a Creative Commons Attribution 4.0 International License. Read Full License 


\section{Abstract}

Sine oculis homeoprotein 1 (SIX1) was discovered to exert an essential role in embryonic development and it was also identified to be re-activated in various types of mammalian cancer. Immunohistochemical and SIX1 gene expression analyses were performed to determine the prognostic role of SIX1 expression. SIX1 expression was suppressed by short hairpin RNA transduction in the SNU398 HCC cell line. The effects of SIX1 on proliferation, epithelial-mesenchymal transition, apoptosis, drug resistance, and sphere formation were assessed in SIX1 knock-down cells. The upregulated expression levels of SIX1 were revealed to be correlated with the stage of the disease in breast, colon and liver cancer, with liver cancer exhibiting the highest expression profile. SIX1 knockdown significantly affected the cell morphology, proliferation, downregulated the protein expression levels of ZEB1, ZEB2 and SNAI1 and upregulated the expression levels of TWIST1 in hepatocellular carcinoma cells. Furthermore, SIX1 knockdown cells were more sensitive to sorafenib treatment; however, the expression profile analysis of the drug resistance genes $A B C B 1, A B C C 1$ and $A B C G 2$ did not explain this sensitivity. Finally, SIX1 knockdown cells were identified to have decreased CD90 levels and lost their sphere-forming ability, which is essential for cancer stem cell properties. Overall, these results indicated that SIX1 expression may be useful as a diagnostic marker for patients with HCC.

\section{Introduction}

Cancer is a major health problem worldwide, demonstrating ever increasing incidence rates [1]. Lung, breast, colorectum and liver cancers are the top four types of cancer responsible for the most cancerrelated deaths [2]. Tumor metastasis, the process by which the primary cancer disseminates to a distant organ and develops into a metastatic lesion, continues to be the primary cause contributing to cancerrelated deaths [3]. Epithelial-mesenchymal transition (EMT) is a fundamental mechanism of cell migration, which has been associated with poor survival and resistance to chemotherapy in numerous types of cancers [4]. The SNAI, TWIST and ZEB (ZEB1 and ZEB2) family of transcription factors are the most thoroughly studied mediators of EMT in human cancers; however, other studies have identified new transcription factors that induced EMT, such as sine oculis homeobox 1 (SIX1) [5]. The overexpression of SIX1 in cancer was first observed in 1998, and it has since been discovered to be associated with a poor prognosis in numerous types of cancer [6]. SIX1 was reported to affect the tumor progression through the regulation of proliferation, invasion and metastasis, genomic instability and the resistance to cell death $[6,7]$. Notably, the EYA2 co-activator was identified to be required for the ability of SIX1 to mediate the prometastatic properties of cells in breast cancer [8]. In addition, SIX1 was previously demonstrated to mediate paclitaxel resistance in breast cancer cells [9]. Drug resistance, a high rate of self-renewal and the ability to differentiate are intrinsic properties of cancer stem cells (CSC), and it has been suggested that EMT and CSC properties are mechanistically related to each other in cells [10]. Interestingly, several recent studies have indicated that SIX1 served an important role in regulating CSC characteristics in leukemia, breast, colorectal, esophageal and pancreatic cancer cells $[11,12,7,13-15]$. 
The present study aimed to determine the expression profile of SIX1 in various types of cancer, in which the highest expression levels were detected in liver cancer. Furthermore, the results revealed that the expression levels of SIX1 were upregulated, which subsequently affected the expression levels of EMT transcription factors, and the drug resistance and self-renewal capacity of Hepatocellular carcinoma (HCC).

\section{Materials And Methods}

\section{Cell Lines and Culture Conditions}

Hepatocellular carcinoma cell lines SNU398, SNU182, SNU475 were cultured in RPMI1640 supplemented with $10 \%$ fetal bovine serum. HCC cell lines HUH7, HEP3B, PLC/PRF/5 and liver cancer cell line HEPG2 were cultured in DMEM supplemented with $10 \%$ fetal bovine serum at $37^{\circ} \mathrm{C}$ in a humidified $5 \% \mathrm{CO}_{2}$ atmosphere. The cell lines were obtained from Dr. Tamer Yagci and were previously used by Yagci et al [16]. All cell lines were routinely checked for mycoplasma contamination. The identities of the cell lines were validated by STR analysis.

\section{Antibodies and Plasmids}

The following antibodies and plasmids were used: anti-ZEB2 antibody (home-made monoclonal antibody, clone 6E5) $[17,18]$, rabbit polyclonal anti-SIX1 antibody (NOVUS,Seattle, WA) and mouse monoclonal SIX1, ZEB1, SNAI1, TWIST1, CK19, VIMENTIN, EYA2, EZRIN, C-PARP, $\beta-A C T I N$, CALNEXIN and ALFA-TUBULIN antibodies (Santa Cruz Biotechnology,Dallas, TX), cell signaling.shRNA lentiviral particles targeting human SIX1 (TRCN0000468669, Sigma-Aldrich, St. Louis, MO, USA), and a non-silencing control, (empty vector, pLK0.1, Addgene \#8453), packaging plasmids pCMV-dR8.2 dvrp (Addgene \#8455) and pCMV-VSV-G (Addgene \#8454).

\section{Production of Lentiviral Particles and Transduction to SNU398 Cell, Generation of SIX1-shRNA and Control Clones}

Lentiviral particles were produced as follows: firstly, lentiviral SIX1 shRNA or control pLKO.1 plasmid was mixed with packaging plasmids and transfection agent PEI (Polysciences, Germany) in Optimem medium (Thermo Fisher Scientific, Rockford, IL, USA). HEK293T was transfected and after 36-48 hours, viral particles were harvested from the supernatant. SNU398 cells transduced with SIX1-shRNA and controlshRNA lentiviral particles in the presence of $8 \mu \mathrm{g} / \mathrm{ml}$ polybrene (\#TR-1003-G, Sigma-Aldrich). $5 \mu \mathrm{g} / \mathrm{ml}$ puromycin (Thermo Fisher Scientific, \#A1113802) was added to the cultures after $24 \mathrm{~h}$ for selecting stable shRNA and control clones.

\section{Proliferation Assay}

The shSIX1-SNU398 and control-SNU398 cells were plated in 96-well plates at an initial density of $5 \times$ 103 cells, with $100 \mu \mathrm{L}$ media per well. After $24 \mathrm{~h}$ of incubation, cell proliferation data were measured at 
12, 24, 36, and 48h by the MTT (3-(4,5-dimethylthiazol-2-yl)-2,5-diphenyltetrazolium bromide) assay (Thermo Fischer Scientific \#M6494).

\section{Western Blotting}

Cells lysed in SDS lysis buffer (10\% glycerol, $2 \%$ SDS in $62.5 \mathrm{mM}$ Tris-HCl) including protease Inhibitor Cocktail (Roche). After the determination of protein concentration, an equal amount of protein from each lysate from was resolved by Sodium dodecyl sulfate-polyacrylamide gel electrophoresis and transferred to a polyvinylidene difluoride membrane (Millipore, \#IPVH00010). Membranes were incubated with primary antibodies at $4^{\circ} \mathrm{C}$ overnight, then were incubated with the secondary antibody for 2 hours at room temperature. Membranes were immersed in SuperSignal West Femto chemiluminescent reagent (Thermo, \#34095) and the protein bands were visualized with the ChemiDocTMXRS system (Bio-Rad, CA).

\section{Gene Expression Analysis}

Total RNA was isolated from shRNA-SNU398 and control-SNU398 with Nucleospin RNA plus isolation kit (Macharey-Nagel, \#740984.250). Complementary DNA was synthesized using the High-Capacity cDNA Reverse Transcription Kit (Applied Biosystems ${ }^{\mathrm{TM}}$, \#4368814) according to the manufacturer's instructions. The real-time quantitative PCR (RT-qPCR) reaction was performed using Maxima SYBR Green qPCR master mix (Thermo Fisher Scientific, \#K0223). Ct values were normalized to GAPDH and calibrated with $\mathrm{Ct}$ of controls. Relative gene expression was calculated by the $\triangle \mathrm{Ct}$ method. Control-SNU398 and shSIX1SNU398 cells were analyzed by RT-qPCR by using the primers listed in Table SI.

\section{Drug Resistance}

Sorafenib (sc220125, Santa Cruz) was prepared according to the manufacturer's instructions. The density of $1.5 \times 10^{5}$ cells/well control-SNU398 and shSIX1-SNU398 were seeded into 6-well plates. $5 \mu \mathrm{M}$ sorafenib was added to the cells when cells reached $70 \%$ confluency. After treatment with sorafenib for 24 hours, cells were harvested, Western blotting and apoptosis analyses were performed. For apoptosis assay, cells were collected resuspended at a density of $1 \times 10^{6}$ cells $/ \mathrm{mL}$ in $1 \mathrm{X}$ binding buffer $(10 \mathrm{mM}$ HEPES/ $\mathrm{NaOH}$ (pH 7.4), $140 \mathrm{mM} \mathrm{NaCl}, 2.5 \mathrm{mM} \mathrm{CaCl}_{2}$ ) and stained with $5 \mu \mathrm{L}$ Annexin V-APC (BD) and $5 \mu \mathrm{L}$ 7AAD-PE (BD) for 15 min at room temperature. Samples were analyzed using Accuri C6 (BD) flow cytometer.

\section{Sphere Formation Assay}

Cells were seeded at a concentration of $1.0 \times 10^{3} \mathrm{cell} / \mathrm{ml}$ into ultra-low attachment surface plates (Corning). These cells were cultured in DMEM (Gibco) supplemented with $50 \mathrm{ng} / \mathrm{ml}$ EGF (R\&D), $10 \mathrm{ng} / \mathrm{ml}$ bFGF (R\&D), $25 \mu \mathrm{g} / \mathrm{ml}$ insulin (Gibco), and $2 \%$ B27 supplement (Gibco) at $37^{\circ} \mathrm{C}$ in a humidified $5 \% \mathrm{CO}_{2}$ atmosphere for 7 days. After incubation, images of spheroids were acquired with a Leica DMi8 inverted microscope, and the diameter of the spheroids was determined. 


\section{Measurement of Cell Surface CD90 Expression with Flow Cytometry}

Cells were resuspended at a density of $3 \times 10^{5} \mathrm{cell} / \mathrm{s} / \mathrm{ml}$ in PBS and transferred into new tubes which were used for the control group and CD90 treated groups. After that, $5 \mu \mathrm{FCR}$ (Miltenyi Biotech) and CD90 (Sony) antibody was added on the tubes and incubated at room temperature samples were analyzed using Accuri C6 (BD) flow cytometer.

\section{Analyses of SIX1 Expression in Human Cancer Samples}

The expression of SIX1 was assessed by RT-qPCR in commercial TissueScan qPCR Cancer Survey cDNA arrays I (Origene, \#CSRT101). The array consists of 72 tumors and 24 non-malignant tissue samples from eight different primary organs (breast, colon, kidney, liver, ovary, thyroid, lung, and prostate) and also provides clinical information (Table S2). SIX1 expression in CDNA arrays was calculated by using the $\Delta \Delta$ Ct method (Log2) [19].

The same tumor types were analyzed in the Expression Project for Oncology (exp0) datasets (GEO accession GSE2109). In this study R software was used for data manipulation, calculation and graphical display (https://www.r-project.org).

Immunohistochemistry was carried out with the Hepatocellular Carcinoma Tissue Array, HLivHCC150PG-01 (Biomax). SIX1 protein expression level was evaluated by integrating the percentage of positive tumor cells and the intensity of positive staining. Briefly, sections were scored as 0 (negative), 1 (bordering), 2 (weak), 3 (moderate) or 4 (strong), whereas the staining extent was scored according to the area percentages: $0(0 \%), 1(1-25 \%), 2(26-50 \%), 3(51 \%-75 \%)$ or $4(76-100 \%)$. The products of staining intensity and extent scores were the final staining scores $(0-16)$. The groups with a score of 0 were excluded from the analyses and did not participate in the statistics.

\section{Statistical Analyses}

GraphPad Prism statistical software was used to analyze the significance of data. Gene expression levels in cell lines and TissueScan cDNA array were represented as mean +SD (standard deviation). UnPaired Student's t-test was used for statistical analyses of data from gene and protein expression experiments in cell lines and tissue array. The differential transcription profile of exp0 datasets was analyzed with Kruskal-Wallis test followed by post-hoc Dunn's test [20]. Significant differences were denoted as follows: ${ }^{\mathrm{P}}<0.05, * * \mathrm{P}<0.01, * * * \mathrm{P}<0.001$.

\section{Results}

\section{SIX1 expression levels are upregulated in various types of cancer}


Previous studies reported that the upregulation of SIX1 expression levels was associated with a poor prognosis in breast, lung, pancreatic, cervical, colorectal and ovarian cancers. Thus, the present study first analyzed the SIX1 expression pattern in liver, colon, breast, ovarian, kidney, lung, thyroid and prostate cancers using cDNA arrays; the array represented tissues from three non-malignant samples and nine tumor samples of eight different cancer types. Compared with the control tissues, a marked upregulation in SIX1 expression levels were observed in the liver, colon, breast, ovary, kidney, lung and prostate tumor tissues, while a slight downregulation was observed in thyroid cancer tissues (Fig. 1a). Interestingly, liver, breast and colon cancer tissues exhibiting high expression levels of SIX1 are very common, indicating that SIX1 upregulation may be associated with cancers with high mortality rates [2]. Thus, the analysis of SIX1 expression levels was further investigated using expression project for oncology (exp0) datasets for tumor tissues of the liver, breast and colon histopathological types, which were grouped according to the pathological stage. The statistical analyses of the colon, liver, breast cancers transcriptional values were analyzed by Kruskal-Wallis test followed by post-doc Dunn's test. Although SIX1 expression was significantly increased in pathological stage 3 and 4 compared to pathological stage 1 in liver and colon cancer samples, a significant increase was detected only in stage 4 compared to stage 1 in breast cancer (Fig. 1b). Following these cDNA array results, the bioinformatics analysis also produced a similar SIX1 expression profile, with the highest expression levels detected in liver cancer.

The upregulation of SIX1 expression levels in liver cancer samples prompted the study into its expression levels with immunohistochemistry (IHC). The tissue array was treated with an anti-SIX1 antibody and analyzed according to the patient grade. Grade 1 and $3 \mathrm{IHC}$ samples had significantly upregulated protein expression levels of SIX1 compared with the non-associated tissues (Fig. 1C). The IHC results also validated the bioinformatics findings. Taken together, these data suggested that the upregulation of the SIX1 mRNA and protein expression levels may be an important indicator of the progression of liver cancer.

\section{SIX1 expression levels are detected in poorly differentiated HCC cell lines and SIX1 knockdown promotes the epithelial phenotype in the SNU398 cell line}

Given the importance of the upregulation of SIX1 in numerous types of cancer, especially liver cancer, the role of the SIX1 transcription factor role in HCC was subsequently investigated. First, SIX1 and EYA2 mRNA expression levels were analyzed in well-differentiated HUH7, HEP3B, HEPG2 and PLC/PRF/5 cell lines and poorly differentiated SNU182, SNU475 and SNU398 liver cancer cell lines. SIX1 transcripts were observed in poorly differentiated cell lines, on the contrary, the EYA2 transcripts, which is required for SIX1 activation [21, 8], was detected in all liver cancer cell lines (Fig. S1).

Western blotting analysis identified that the poorly differentiated cell lines expressed the SIX1 protein, especially in SNU475, SNU398 and SNU182 cells. According to our Western blot result, SIX1 protein may be present in SNU 475 in the hyperphosphorylated state, causing it to lose its ability to bind DNA [22]. For this reason, SNU398 cells, which had the second highest SIX1 expression levels, were selected for further analysis (Fig. 2a). SIX1-shRNA-SNU398 (shSIX1-SNU398) and plKo.1-shRNA-SNU398 (control-SNU398) 
stable clones were generated to determine the role of SIX1 in HCC cells (Fig. 2b). Changes in the SIX1 expression levels were identified to promote marked differences in the cellular morphology; for example, compared with the control-SNU398 clones, shSIX1-SNU398 cells exhibited a clustered distribution, which is an indicator of an epithelial phenotype (Fig. 2c). In order to explore the effects of SIX1 knockdown on the proliferation capacity, we analyzed shSIX1-SNU398 and control-SNU398 HCC cell clones cell proliferation by MTT assay. Our results from cell viability assays were in line with previous studies [23, 24], demonstrating that cellular proliferation is significantly inhibited in shSIX1-SNU398 at 24, 36 and 48 hours compared to control-SNU398 cells (Fig. 2 d)

\section{Knockdown of SIX1 expression affects the expression levels of EMT markers}

Subsequently, the effect of SIX1 knockdown on ZEB1, ZEB2, TWIST, EPCAM, VIM, CDH1, SOX2, ITGA5 and ITGB1 expression levels was analyzed using RT-qPCR. Previous studies indicated that SIX1 promoted the a5 31 integrin-mediated metastatic capability of cervical cancer cells, and SIX1 was discovered to bind to the regulatory element of SOX2 and control SOX2 expression levels in human glioma cell lines $[25,26]$. Thus, whether SIX1 could affect the expression levels of EMT transcription factors and EMT markers in HCC was investigated. The results obtained in the present study were consistent with previous reports; for example, ITGA5, ITGB1 and SOX2 expression levels were discovered to be downregulated by $\sim 0.4$-fold in the SIX1 knockdown clones. The expression levels of ZEB1, ZEB2 and TWIST1 were downregulated in shSIX1-SNU398 cells compared to control clones. In addition, the results revealed that the expression levels of VIM, which is a mesenchymal marker, were downregulated, while the expression levels of EPCAM, which is an epithelial marker, were upregulated in shSIX1-SNU398 clones (Fig. 3a).

The western blotting results were consistent with the RT-qPCR data, suggesting that SIX1 may serve a role in the downregulation of ZEB1, ZEB2, SNAI1 and vimentin expression levels, which are EMT transcription factors. Interestingly, TWIST1 expression levels were downregulated at the mRNA level, while its protein expression levels were upregulated. However, the CK19 epithelial marker was significantly upregulated in the shSIX1-SNU398 clone. Interestingly, SIX1 silencing had no significant effect on EYA2 expression at the protein level. The suppression of SIX1 expression also significantly downregulated the expression levels of the EZRIN protein, which is transcriptionally regulated by SIX1 and regulates cytoskeletal organization and adhesion [27] (Fig. 3b).

\section{SIX1 knockdown upregulates c-PARP expression levels and the apoptotic rate following sorafenib treatment in HCC cells}

EMT is a possible mechanistic basis for anti-cancer drug resistance [28]. Until now, to the best of our knowledge, the SIX1-dependent effects on drug treatment has only been investigated in gastric and breast cancer cells $[9,29]$. To further determine the effect of SIX1 in HCC, shSIX1 and control cell clones were treated with sorafenib, which is a standard chemotherapeutic drug for HCC. To understand the effect of SIX1 knockdown on the drug resistance, flow cytometric analysis was performed and it was noted that SIX1 knockdown cells were more sensitive to sorafenib treatment. The apoptotic cell number in shSIX1 and control cell clones was $93.9 \%$ and $66.7 \%$, respectively, and the knockdown of SIX1 
expression significantly increased the number of apoptotic cells (Fig. 4a). Moreover, these findings were further confirmed by the subsequent protein changes. After exposure to sorafenib, the expression of cleaved PARP was increased in shSIX1-SNU398 cells compare to control-SNU398 and untreated cells (Fig. 4b). These results indicated that SIX1 expression may exert a significant influence on the chemotherapeutic drug response.

There are various molecular mechanisms involved in multidrug resistance, and ATP-binding cassette (ABC) transporters are a family of transporter proteins that are responsible for drug resistance [30]. Therefore, whether SIX1 expression exerted an effect on the expression levels of the drug resistance genes, $A B C G 2, A B C C 1$ and $A B C B 1$, was investigated. Interestingly, the expression levels of $A B C C 1$ and $A B C B 1$ did not markedly change, while the expression levels of $A B C G 2$ were markedly upregulated (Fig.

4c). However, this finding did not fully explain the role of SIX1 in the drug sensitivity mechanism. Furthermore, the role of SIX1 knockdown on the drug response and the expression levels of drug resistance-related genes were analyzed. SIX1 knockdown cells were more sensitive to sorafenib; however, the expression levels of drug-resistant associated genes were not changed or downregulated.

\section{SIX1 expression levels are important for HCC cell clonogenicity capacities}

CSCs have several biological properties that distinguish them from other cancer cells, such as resistance to treatment, evasion from cell death, dormancy and a self-renewal capacity [31]. To determine the role of SIX1 on the self-renewal capacity of HCC cell clones, a sphere formation assay was performed. shSIX1SNU398 and control-SNU398 cells were capable of forming morphological spheres; however, the spheres of SIX1 knockdown cells were significantly reduced both the size and number of spheres formed. shSIX1SNU398 sphere colony $(>200 \mu \mathrm{m})$ and control-SNU398 colony $(<65 \mu \mathrm{m})$ spheres are depicted in Fig. 5a. The control cells formed significantly increased numbers of spheres $(>100 \mu \mathrm{m})$ compared with the SIX1 knockdown cells. Notably, following the second passage of sphere cells, shSIX1-SNU398 cell spheres did not retain the ability to self-renewal (Fig. 5b).

To validate the morphological data, the mRNA expression levels of $S I X 1, Z E B 2, V I M, K L F 4$ and THY1 were analyzed in the control and SIX1 knockdown sphere-forming cells. The results revealed that ZEB2, VIM, KLF4 and THY1 gene expression levels were significantly downregulated in SIX1 knockdown sphere cells (Fig. 5c).

A recent study demonstrated that the CSC marker CD90, a THY1 gene product, was important for the cell cycle, migration, invasion and sphere forming ability of HCC cells [32]. The observed SIX1-dependent effects in the self-renewal capacity prompted the analysis of the CD90 cell population in shSIX1-SNU398 and control-SNU398 cells by flow cytometry; the knockdown of SIX1 significantly decreased the CD $90^{+}$ subpopulation in shSIX1-SNU398 cell clones (65.5\%) compared with the control-SNU398 cell clones (35.1\%) (Fig. 5d).

\section{Discussion}


Hepatocellular carcinoma (HCC) is the most common type of liver cancer, demonstrating a high mortality rate. Several studies have reported that the expression levels of SIX1 were upregulated in various types of mammalian cancer and that the overexpression of SIX1 lead to increased motility of cancer cells, including in breast, colorectal and hepatocellular carcinomas [33, 7, 34-40, 1]. In the present study, SIX1 expression levels were discovered to be upregulated in the different sub-types of cancer samples, except thyroid cancer in CDNA arrays analyses. A recent paper pointed out SIX1 level was positively associated with tumor size and lymph node metastasis in thyroid cancer [41]. Unfortunately, we tested thyroid expression profile in just cDNA array includes; 3 non-malignant, 6 papillary, 1 hurtle cell, 1 medullary (metastatic) carcinoma histologic types cancer tissues (Table S1) and the Ct value of metastatic thyroid sample was early. Future studies will be explained to more detailed the SIX1 expression role in thyroid cancer. The up-regulated SIX1 expression was detected especially in the liver, colon, and breast cancer tissues compared with the non-malignant tissues in cDNA analyses. The present study further explored the expression of SIX1 in the expO dataset according to the patient stage in three types of cancer (liver, colon and breast); interestingly, the expression levels of SIX1 were upregulated according to the patient stage. Thus, the upregulation of SIX1 expression levels appears to be important for liver, colon and breast cancers, which are all placed in the top five cancers responsible for cancer-related mortalities [2]. The findings of the present study are consistent with previous studies, and the reported SIX 1 overexpression in our in-silico and in-vivo data suggested that SIX1 may be important for HCC progression.

Resistance to chemotherapy agents, limited available treatment options and high mortality rates are associated with the incidence of HCC, and prompted the present study to investigate the role of SIX1 in hepatocarcinogenesis in more detail. The results revealed that the SIX1 expression patterns were distinct in well and poorly differentiated HCC cell lines. Therefore, the expression levels of SIX1 in the SNU398 cell line, which naturally had high SIX1 expression levels, were knocked down, which promoted the downregulated of the expression levels of the mesenchymal marker Vimentin and an upregulation in the expression levels of epithelial markers, such as CK19 and EPCAM in cells. In addition, in accordance with these results, the cell morphology dramatically changed. The present study initially hoped to perform wound healing and colony-forming assays; however, these assays were not performed because of the changes in cell morphology were dependent on SIX1 suppression. SIX1 has been reported to control the expression of various genes that have significant roles in cancer progression, such as EZRIN in rhabdomyosarcoma, SOX 2 in glioma and a5 $\beta 1$ integrin in cervical cancer cells [38, 26, 25]. Consistent with these findings, the present study observed a decrease in the expression levels of EZRIN, SOX2, ITGA5 and ITGB1 in SIX1 knockdown cells. In addition, SIX1-dependent protein expression levels analysis revealed that the expression levels of the epithelial marker CK19 were significantly upregulated, while those of the mesenchymal marker Vimentin was markedly downregulated in HCC. Conversely, the expression levels of TWIST1 were significantly upregulated at the protein levels. Interestingly, EMT transcription factor analysis also revealed that the expression levels of the ZEB1, ZEB2 and SNAI1 transcription factors were significantly downregulated. In contrast to our results, the changes in the expression levels of the EMT markers ZEB1, ZEB2, SNAI2, TWIST1, TWIST2 and VIM in SIX1 overexpressing esophageal squamous carcinoma cell clones were not investigated [15]. 
The link between EMT and drug resistance prompted further investigations into the role of SIX1 in drug resistance. In the present study, SIX1 knockdown cells were discovered to be more sensitive to sorafenib treatment and influenced the c-PARP protein expression levels and apoptotic cell levels. SIX1 overexpression conferred resistance in breast cancer, while the suppression of SIX1 increased the sensitivity for a drug in gastric cancer cells $[9,29]$. Transcription factors of the EMT process are known to mediate ATP binding cassette transporters, which have a role in the drug resistance mechanism [42]. Despite SIX1 knockdown cells being more sensitive to sorafenib treatment, the expression profiles of the $A B C$ transporter members $A B C B 1, A B C C 1$ and $A B C G 2$ did not explain the drug response. A possible explanation for this discrepancy may be that another signal transduction mechanism was involved in this process. For instance, a previous study reported that the NF-KB signaling pathway, which serves a crucial role in the development of drug resistance, was activated by SIX1 and SIX2 [43, 44].

Cancer stem cells share numerous characteristics with normal stem cells, including their differentiation and self-renewal abilities, and their drug resistance properties [45]. Previous studies have demonstrated that the SIX1 transcription factor affected the properties of CSCs in a variety of mammalian cancer cells, except for in HCC. Therefore, after determining that SIX1 served a role in the drug resistance mechanism, the present study investigated the role of SIX1 on the self-renewal ability of HCC cells. The results revealed that fewer spheres with smaller diameters were formed in SIX1 knockdown cell clones compared with the control cell line, and more importantly, after the second passage, no sphere formation was observed. The gene expression analysis of spheres formed from the SIX1 knockdown cell line, the mRNA expression levels of $Z E B 2, V I M, K L F 4$ and $T H Y 1$ were markedly downregulated compared with the control cell spheres. KLF4 transcription factor expression decreased in SIX1 knockdown spheres and a recent study pointed out the KLF4 transcription factor can induce a CSC-like phenotype in HCC [46]. Zhang et al [32] revealed that CD90, a gene product of THY1 and a marker of HCC CSCs, promoted the sphereforming ability in HCC. The results of the present study revealed that SIX1 suppression affected the CD90 positive cell count and the sphere-forming ability of the cells in HCC. Thus, these findings suggested that SIX1 may be used as a biomarker for liver cancer progression and may serve crucial roles in the drug resistance mechanism and cell renewal capacity in hepatocarcinogenesis. The future studies will be needed to tested SIX1-dependent tumor stemness capacity in in vivo models of HCC and also additional studies will be required to assess EMT markers protein expression profile in SIX1-dependent HCC cell colonies.

Although the SIX1 protein was also expressed in the SNU475 and SNU182 cell lines, the present study only used the SNU398 cell line for further investigations, because the SIX1 protein is found in the hyperphosphorylated form in SNU475 cells, and a previous study indicated that the hyperphosphorylated form of the SIX1 protein had no DNA binding capacity [22]. The other HCC cell line, SNU182, only had slightly increased SIX1 expression levels, so it was not used to determine the SIX1-mediated effects in HCC cells. Notably, SIX1 silencing exerted no significant effect on the protein expression levels of EYA2, a SIX1 co-activator. These results indicated that the observed findings in the present study were through SIX1, not via EYA2. 
In conclusion, the present study provided further information on the functional roles of SIX1 in hepatocarcinogenesis and also demonstrated the importance of SIX1, both as a biomarker and as a target for drug treatment.

\section{Declarations}

\section{Conflict of Interest}

The authors declare that they have no conflict of interest.

\section{Author's contributions:}

P.B-E. designed the study. P.B-E., A.A., N.S. and E.E. performed the experiments. P.B-E., A.A., N.S. and E.E. analyzed and interpreted the data. P.B-E, A.A. and N.S wrote the manuscript. All authors read and approved the final manuscript.

\section{Acknowledgments}

The authors thank Dr. Tamer Yagci for his valuable support.

\section{References}

1. Global Burden of Disease Cancer C, Fitzmaurice C, Akinyemiju TF, Al Lami FH, Alam T, AlizadehNavaei R et al (2018) Global, Regional, and National Cancer Incidence, Mortality, Years of Life Lost, Years Lived With Disability, and Disability-Adjusted Life-Years for 29 Cancer Groups, 1990 to 2016: A Systematic Analysis for the Global Burden of Disease Study. JAMA Oncol. 4(11):1553-1568. doi:10.1001/jamaoncol.2018.2706

2. Bray F, Ferlay J, Soerjomataram I, Siegel RL, Torre LA, Jemal A (2018) Global cancer statistics 2018: GLOBOCAN estimates of incidence and mortality worldwide for 36 cancers in 185 countries. CA: a cancer journal for clinicians 68(6):394-424

3. Liu Q, Zhang H, Jiang X, Qian C, Liu Z, Luo D (2017) Factors involved in cancer metastasis: a better understanding to "seed and soil" hypothesis. Molecular cancer 16(1):176

4. Singh A, Settleman J (2010) EMT, cancer stem cells and drug resistance: an emerging axis of evil in the war on cancer. Oncogene 29(34):4741

5. Goossens S, Vandamme N, Van Vlierberghe P, Berx G (2017) EMT transcription factors in cancer development re-evaluated: beyond EMT and MET. Biochimica et Biophysica Acta (BBA)-Reviews on Cancer. 1868(2):584-91

6. Blevins MA, Towers CG, Patrick AN, Zhao R, Ford HL (2015) The SIX1-EYA transcriptional complex as a therapeutic target in cancer. Expert opinion on therapeutic targets 19(2):213-225

7. Lerbs T, Bisht S, Schölch S, Pecqueux M, Kristiansen G, Schneider M et al (2017) Inhibition of Six1 affects tumour invasion and the expression of cancer stem cell markers in pancreatic cancer. BMC 
cancer 17(1):249

8. Farabaugh SM, Micalizzi DS, Jedlicka P, Zhao R, Ford HL (2012) Eya2 is required to mediate the prometastatic functions of Six 1 via the induction of TGF- $\beta$ signaling, epithelial-mesenchymal transition, and cancer stem cell properties. Oncogene 31(5):552

9. Li Z, Tian T, Hu X, Zhang X, Nan F, Chang Y et al (2013) Six1 mediates resistance to paclitaxel in breast cancer cells. Biochem Biophys Res Commun 441(3):538-543

10. Yang M, Liu P, Huang P (2016) Cancer stem cells, metabolism, and therapeutic significance. Tumor Biology 37(5):5735-5742

11. Chu Y, Chen Y, Li M, Shi D, Wang B, Lian Y et al (2019) Six1 regulates leukemia stem cell maintenance in acute myeloid leukemia. Cancer Science

12. Kingsbury TJ, Kim M, Civin Cl (2019) Regulation of cancer stem cell properties by SIX1, a member of the PAX-SIX-EYA-DACH network. Advances in cancer research 141:1-42

13. Iwanaga R, Wang C-A, Micalizzi DS, Harrell JC, Jedlicka P, Sartorius CA et al (2012) Expression of Six 1 in luminal breast cancers predicts poor prognosis and promotes increases in tumor initiating cells by activation of extracellular signal-regulated kinase and transforming growth factor-beta signaling pathways. Breast Cancer Res 14(4):R100

14. Xu H, Zhang Y, Peña MM, Pirisi L, Creek KE (2017) Six1 promotes colorectal cancer growth and metastasis by stimulating angiogenesis and recruiting tumor-associated macrophages. Carcinogenesis 38(3):281-292

15. Nishimura T, Tamaoki M, Komatsuzaki R, Oue N, Taniguchi H, Komatsu M et al (2017) SIX 1 maintains tumor basal cells via transforming growth factor- $\beta$ pathway and associates with poor prognosis in esophageal cancer. Cancer science 108(2):216-225

16. Yalim-Camci I, Balcik-Ercin P, Cetin M, Odabas G, Tokay N, Sayan AE et al (2019) ETS1 is coexpressed with ZEB2 and mediates ZEB2-induced epithelial-mesenchymal transition in human tumors. 58(6):1068-81

17. Sayan AE, Griffiths TR, Pal R, Browne GJ, Ruddick A, Yagci T et al (2009) SIP1 protein protects cells from DNA damage-induced apoptosis and has independent prognostic value in bladder cancer. Proceedings of the National Academy of Sciences. 106(35):14884-9

18. Oztas E, Avci ME, Ozcan A, Sayan AE, Tulchinsky E, Yagci T (2010) Novel monoclonal antibodies detect Smad-interacting protein 1 (SIP1) in the cytoplasm of human cells from multiple tumor tissue arrays. Exp Mol Pathol 89(2):182-189

19. Avci ME, Konu O, Yagci T (2008) Quantification of SLIT-ROBO transcripts in hepatocellular carcinoma reveals two groups of genes with coordinate expression. BMC Cancer 8(1):392

20. Kammerer S, Sokolowski A, Hackl H, Platzer D, Jahn SW, El-Heliebi A et al (2016) KCNJ3 is a new independent prognostic marker for estrogen receptor positive breast cancer patients.;7(51):84705

21. Ford HL, Kabingu EN, Bump EA, Mutter GL, Pardee AB (1998) Abrogation of the G2 cell cycle checkpoint associated with overexpression of HSIX1: a possible mechanism of breast carcinogenesis. Proceedings of the National Academy of Sciences.95(21):12608-13 
22. Ford HL, Landesman-Bollag E, Dacwag CS, Stukenberg PT, Pardee AB, Seldin DCJJoBC. (2000) Cell cycle-regulated phosphorylation of the human SIX1 homeodomain protein. 275(29):22245-54

23. Li Z, Tian T, Hu X, Zhang X, Li L, Nan F et al (2014) Targeting Six1 by lentivirus-mediated RNA interference inhibits colorectal cancer cell growth and invasion. 7(2):631

24. Li Z, Tian T, Lv F, Chang Y, Wang X, Zhang L et al (2013) Six1 promotes proliferation of pancreatic cancer cells via upregulation of cyclin D1 expression. 8(3):e59203

25. Liu D, Zhang X-X, Wan D-Y, Xi B-X, Ma D, Wang H et al (2014) Sine oculis homeobox homolog 1 promotes a $5 \beta 1$-mediated invasive migration and metastasis of cervical cancer cells. Biochemical biophysical research communications 446(2):549-554

26. De Lope C, Martín-Alonso S, Auzmendi-Iriarte J, Escudero C, Mulet I, Larrasa-Alonso J et al (2019) SIX1 represses senescence and promotes SOX2-mediated cellular plasticity during tumorigenesis. Scientific reports 9(1):1412

27. Yu Y, Davicioni E, Triche TJ, Merlino G (2006) The homeoprotein six1 transcriptionally activates multiple protumorigenic genes but requires ezrin to promote metastasis. Cancer research 66(4):1982-1989

28. Shibue T, Weinberg RA (2017) EMT, CSCs, and drug resistance: the mechanistic link and clinical implications. Nature reviews Clinical oncology 14(10):611

29. Du P, Zhao J, Wang J, Liu Y, Ren H, Patel R et al (2017) Sine Oculis Homeobox Homolog 1 regulates mitochondrial apoptosis pathway via Caspase-7 in gastric cancer cells. Journal of Cancer 8(4):636

30. Choi C-H (2005) ABC transporters as multidrug resistance mechanisms and the development of chemosensitizers for their reversal. Cancer cell international 5(1):30

31. Hu J, Mirshahidi S, Simental A, Lee SC, De Andrade Filho PA, Peterson NR et al (2019) Cancer stem cell self-renewal as a therapeutic target in human oral cancer. Oncogene.:1

32. Zhang K, Che S, Su Z, Zheng S, Zhang H, Yang S et al (2018) CD90 promotes cell migration, viability and sphere-forming ability of hepatocellular carcinoma cells. International journal of molecular medicine 41(2):946-954

33. Behbakht K, Qamar L, Aldridge CS, Coletta RD, Davidson SA, Thorburn A et al (2007) Six1 overexpression in ovarian carcinoma causes resistance to TRAIL-mediated apoptosis and is associated with poor survival. Cancer research 67(7):3036-3042

34. Ono H, Imoto I, Kozaki K, Tsuda H, Matsui T, Kurasawa Y et al (2012) SIX1 promotes epithelialmesenchymal transition in colorectal cancer through ZEB1 activation. Oncogene 31(47):4923

35. Sehic D, Karlsson J, Sandstedt B, Gisselsson D (2012) SIX1 protein expression selectively identifies blastemal elements in Wilms tumor. Pediatric blood cancer 59(1):62-68

36. Tan J, Zhang C, Qian J (2011) Expression and significance of Six1 and Ezrin in cervical cancer tissue. Tumor Biology 32(6):1241

37. Wang C-A, Jedlicka P, Patrick AN, Micalizzi DS, Lemmer KC, Deitsch E et al (2012) SIX1 induces lymphangiogenesis and metastasis via upregulation of VEGF-C in mouse models of breast cancer. 
The Journal of clinical investigation 122(5):1895-1906

38. Yu Y, Khan J, Khanna C, Helman L, Meltzer PS, Merlino G (2004) Expression profiling identifies the cytoskeletal organizer ezrin and the developmental homeoprotein Six-1 as key metastatic regulators. Nature medicine 10(2):175

39. Ng K, Man K, Sun C, Lee T, Poon R, Lo C et al (2006) Clinicopathological significance of homeoprotein Six1 in hepatocellular carcinoma. British journal of cancer 95(8):1050

40. Ng KT, Lee TK, Cheng Q, Wo JY, Sun CK, Guo DY et al (2010) Suppression of tumorigenesis and metastasis of hepatocellular carcinoma by shRNA interference targeting on homeoprotein Six1. International journal of cancer 127(4):859-872

41. Yang C, Xu W, Gong J, Chai F, Cui D, Liu ZJO et al (2020) Six1 Overexpression Promotes Glucose Metabolism and Invasion Through Regulation of GLUT3, MMP2 and Snail in Thyroid Cancer Cells. 13:4855-63

42. Saxena M, Stephens MA, Pathak H, Rangarajan A (2011) Transcription factors that mediate epithelial-mesenchymal transition lead to multidrug resistance by upregulating $A B C$ transporters. Cell death disease 2(7):e179

43. Liu Z, Mar KB, Hanners NW, Perelman SS, Kanchwala M, Xing C et al (2019) A NIK-SIX signalling axis controls inflammation by targeted silencing of non-canonical NF-KB. Nature. 1

44. Godwin P, Baird A-M, Heavey S, Barr M, O'Byrne K, Gately KA (2013) Targeting nuclear factor-kappa B to overcome resistance to chemotherapy. Frontiers in oncology 3:120

45. Lobo NA, Shimono Y, Qian D, Clarke MF (2007) The biology of cancer stem cells. Annu Rev Cell Dev Biol 23:675-699

46. Firtina Karagonlar Z, Akbari S, Karabicici M, Sahin E, Tercan Avci S, Ersoy N et al (2020) A Novel Function for KLF4 in Modulating the De-Differentiation of EpCAM-/CD133 - nonStem Cells into EpCAM+/CD133 + Liver Cancer Stem Cells in HCC Cell Line HuH7. 9(5):1198

\section{Figures}


a

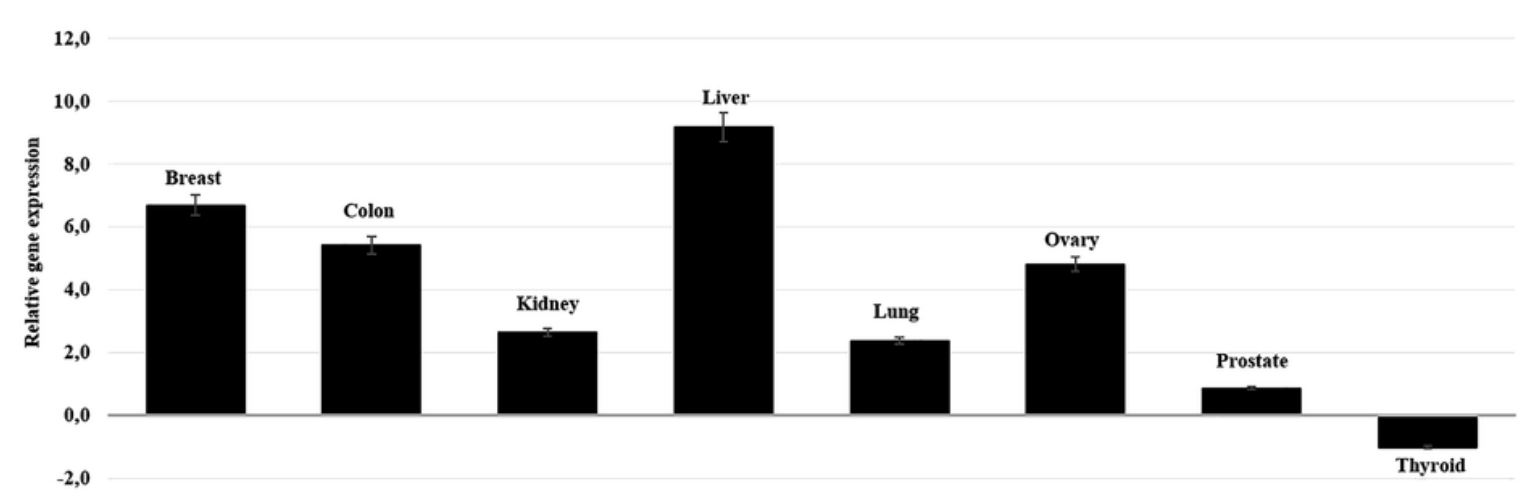

b
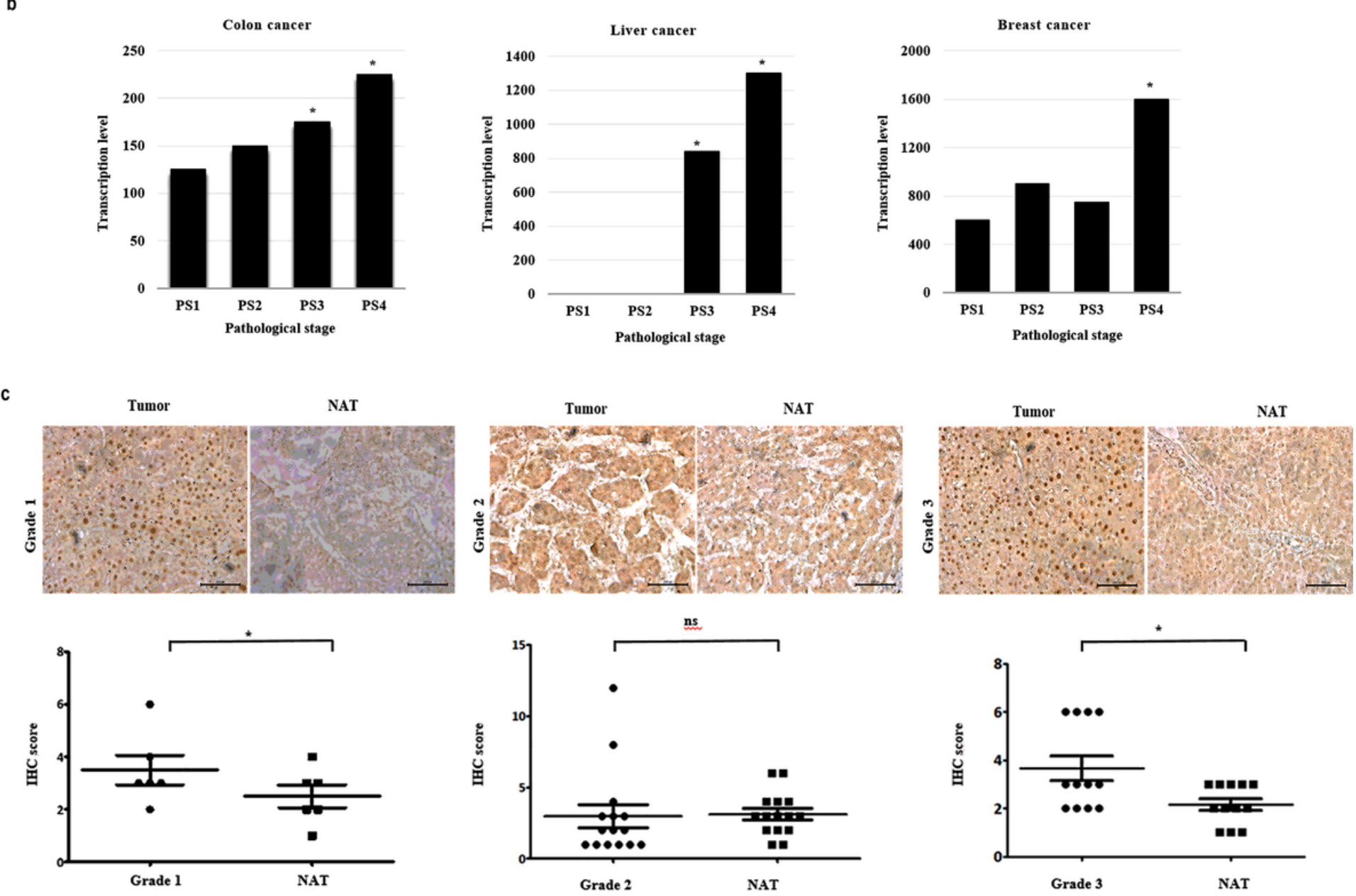

Figure 1

SIX1 gene expression levels both in vivo and using bioinformatic analysis in different types of cancer. a Analysis of the expression levels of SIX1 in cancer CDNA arrays representing tissues from three nonmalignant and nine tumor samples from eight different types of cancer. Compared with the control tissue, the expression levels of SIX1 in the liver, colon, breast, ovary, kidney, lung and prostate tumor tissues were markedly upregulated, while they were slightly downregulated in thyroid cancer. mRNA expression levels were quantified using the 2- $\Delta \Delta \mathrm{Cq}$ method. b mRNA transcription levels of SIX1 in colon, liver and breast cancers depending on the pathological stages using an Expression Project for Oncology (exp0) dataset. 
SIX1 expression was significantly upregulated in pathological stage 3 compared to pathological stage 1 in liver and colon cancer, and also SIX1 high expression was detected in stage 4 compared to stage 1 in all 3 types of cancer $\left({ }^{*} P<0.05\right)$. c SIX1 expression levels in tumor tissues were compared with normal tissues in three different stages of hepatocellular carcinoma. SIX1 expression levels in grade $1(n=6)$ and grade $3(n=20)$ were significantly different in the tumor tissues compared with the normal tissues. In contrast, no significant differences were observed in grade 2 tissues $(n=12)$. NAT, non-associated tissue. $* P<0.05$, unpaired Student's t-test. Magnification, $x 40 ;$ scale bar, $200-\mu m$.

a

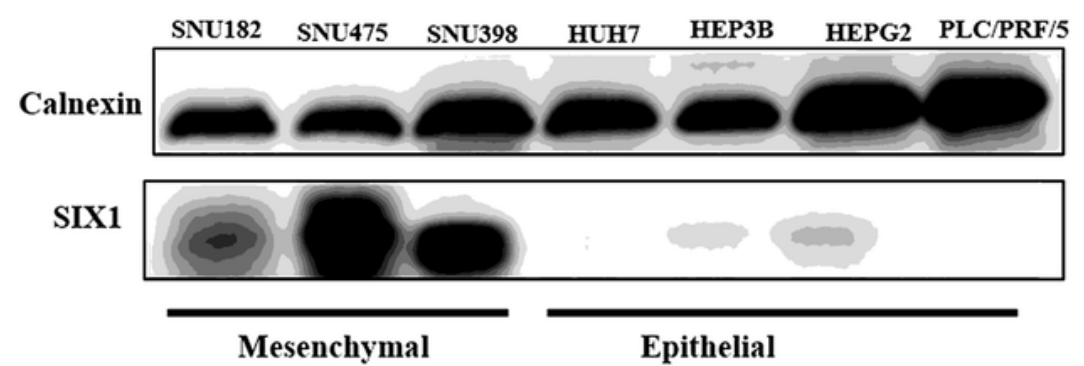

b

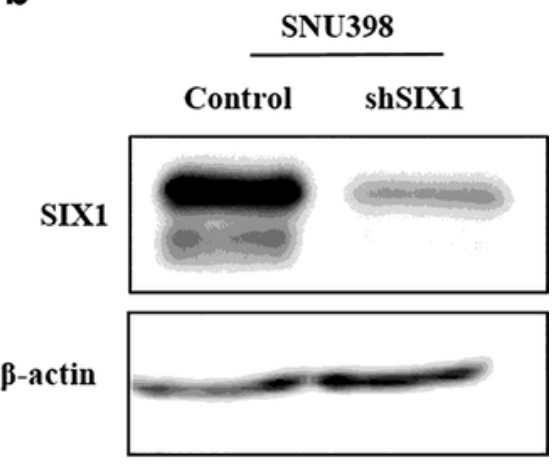

C

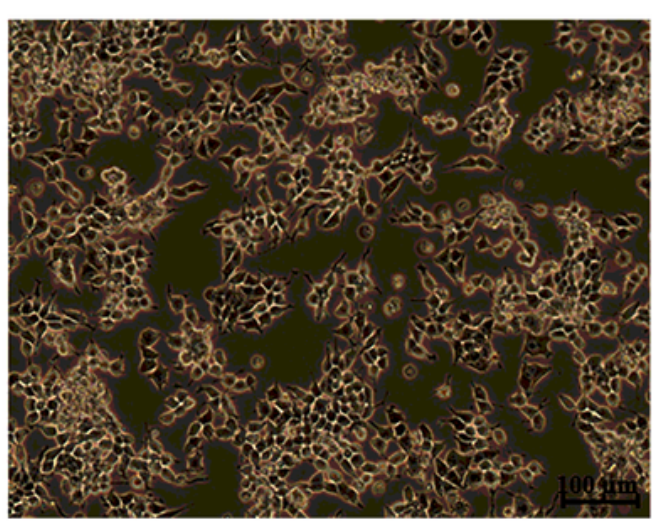

Control-SNU398

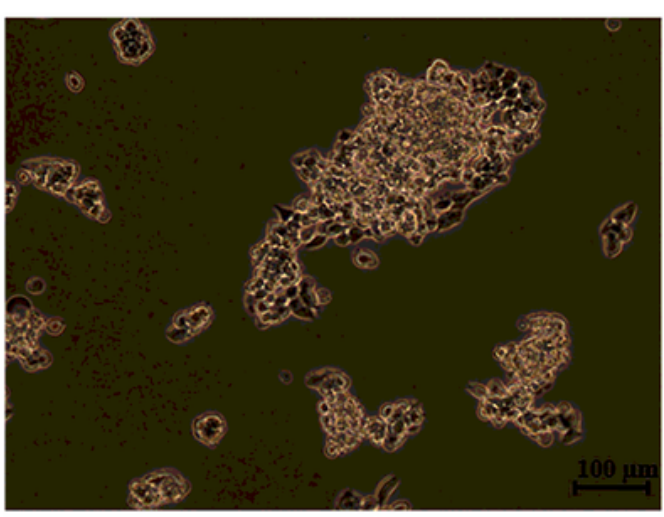

shSIX1-SNU398

d

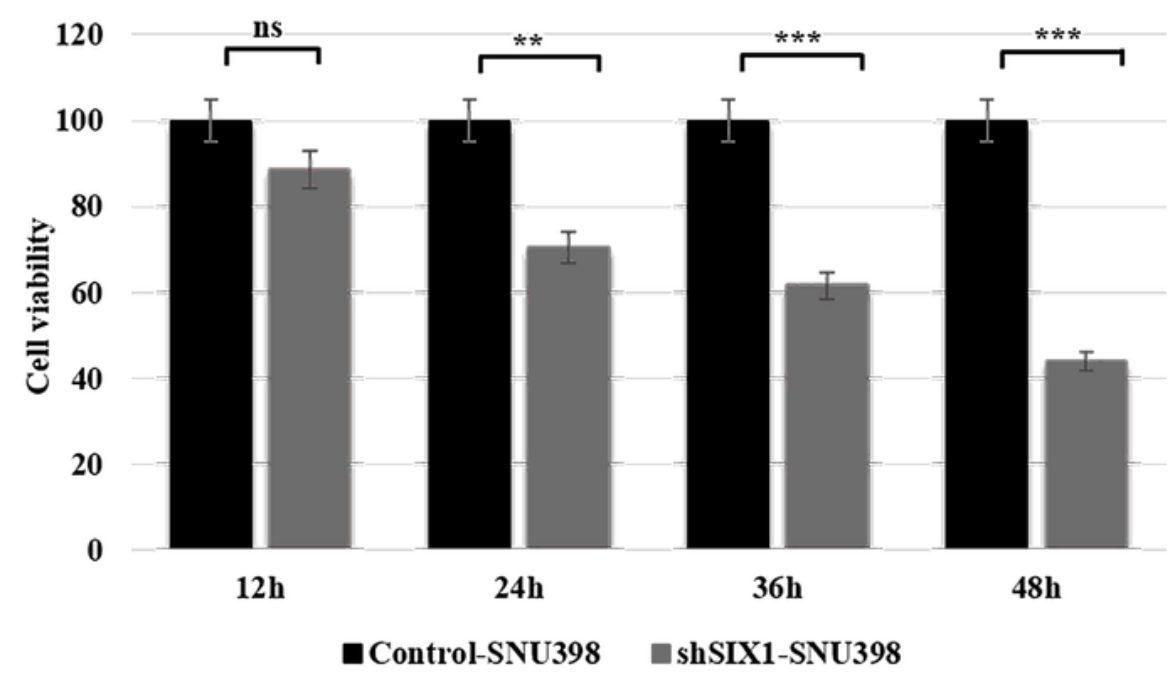


Figure 2

Expression levels of SIX1 in HCC cells and knockdown of SIX1 expression levels in the SNU398 cell line. a Expression levels of SIX1 in different HCC cell lines with mesenchymal and epithelial characteristics. $b$ Transfection efficiency of the knockdown of the SIX1 gene in the SNU398 cell line was validated using western blotting. $\beta$-actin was used as the loading control. c Inhibition of SIX1 expression levels in the SNU398 cell line initiated a change in the cell morphology. The different cell morphologies of the shSIX1SNU398 and control-SNU398 cells were observed under an inverted microscope. Magnification, x20; scale bar, 100- $\mu \mathrm{m}$. (D) Bars represent proliferation of cells with and without SIX1 expression as determined by MTT assay. The differences between groups were analyzed using a Student's t-test. ns, non-significant. $\star \star \star * P<0.001,{ }^{* \star *} \mathrm{P}<0.0001$.

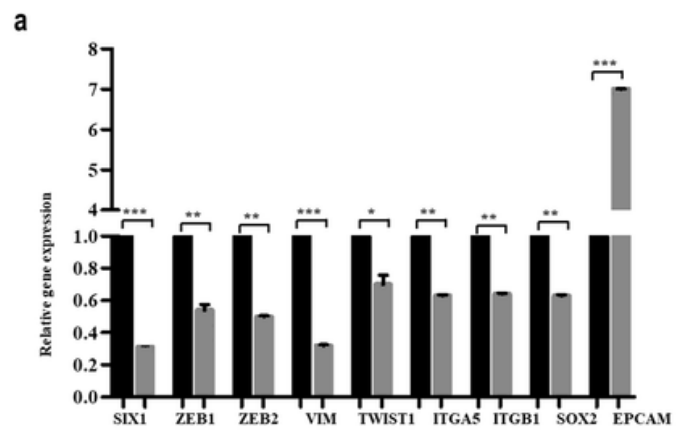

b
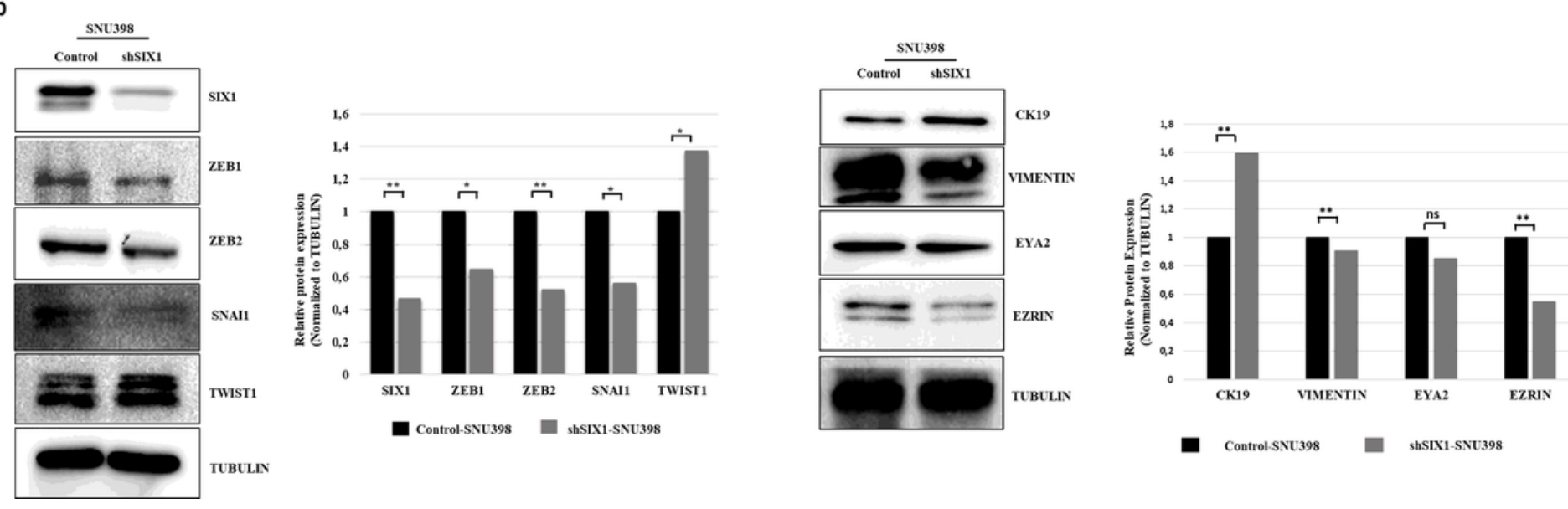

Figure 3

SIX1 knockdown regulates EMT mRNA and protein expression levels. a Effect of SIX1 knockdown on the expression levels of ZEB1, ZEB2 and EMT-related genes was analyzed using RT-qPCR. b Expression levels of EMT-related proteins in the control-SNU398 and shSIX1-SNU398 cells were analyzed using western blotting. a-tubulin served as the loading control. The differences between the groups were analyzed using a Student's t-test. ${ }^{*} P<0.05,{ }^{*} P<0.001, * \star * P<0.0001$. 
a

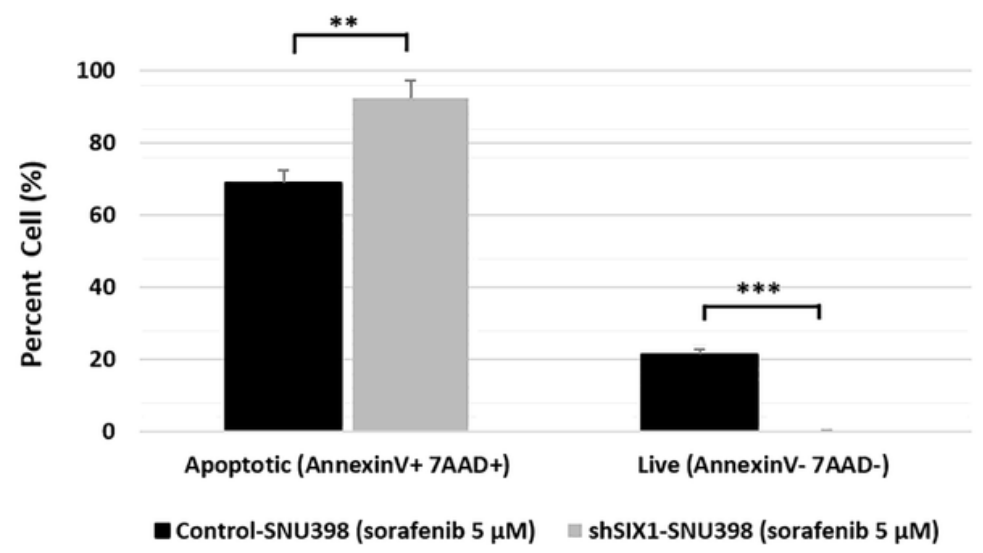

b

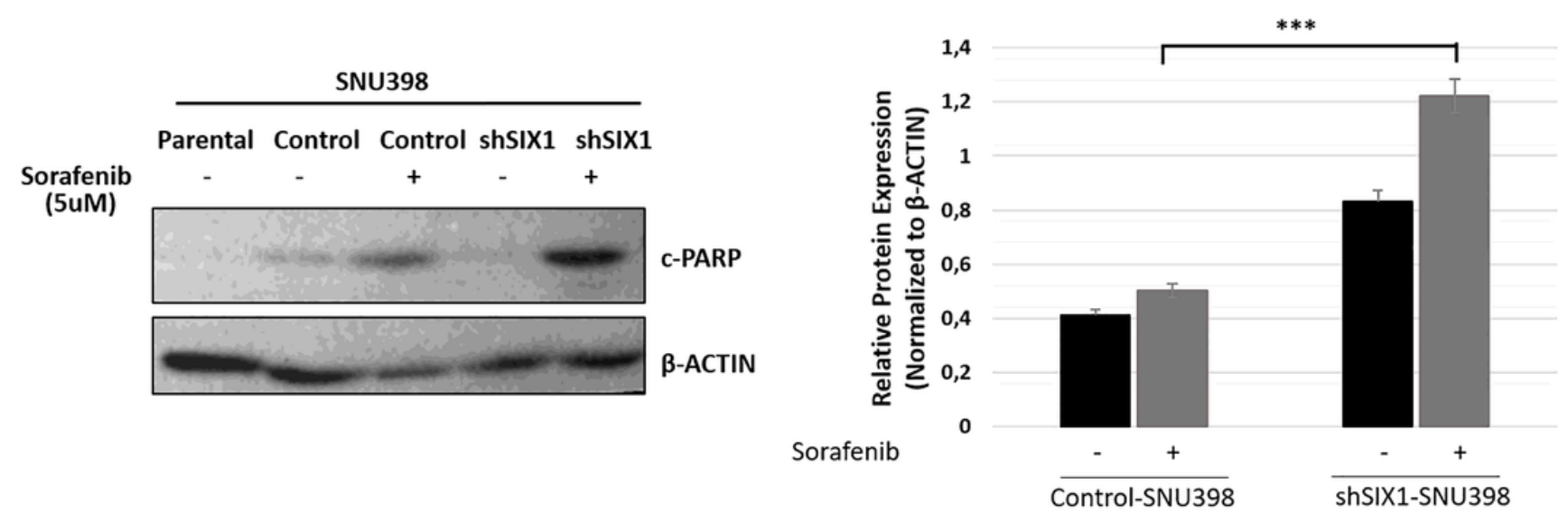

C

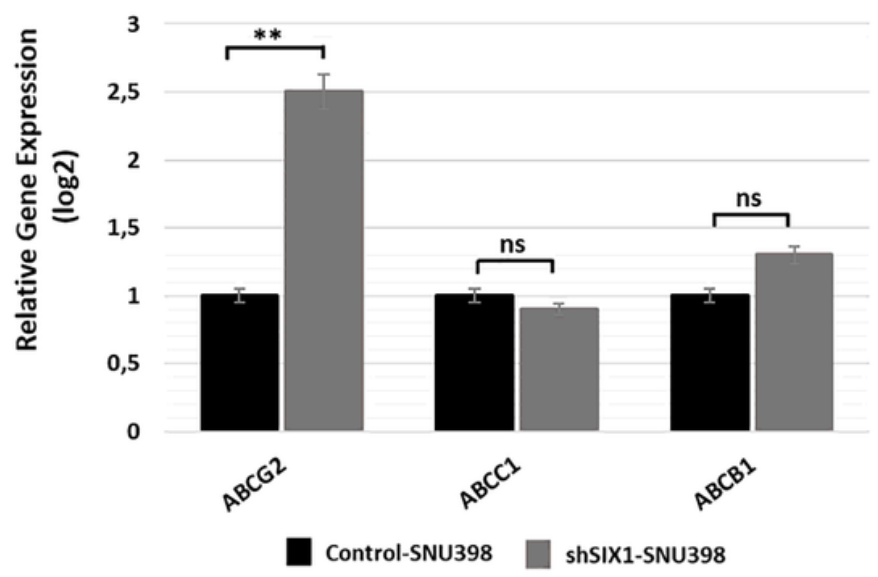

Figure 4

Effect of SIX1 knockdown on apoptosis and drug resistance in the presence of sorafenib. a The percentage of apoptotic and living cells are shown. Apoptotic cells were analyzed by a BD Accuri C6 flow cytometer. b Western blotting was used to investigate the expression levels of the c-PARP protein in SNU398 (parental), shSIX1-SNU398 and control-SNU398 cells. The c-PARP levels was increased in shSIX1-SNU398 cells compared to control-SNU398 cells presence of $5 \mu \mathrm{M}$ sorafenib. $\beta$-actin was used as the loading control. c Effect of SIX1 knockdown in the presence of $5 \mu \mathrm{M}$ sorafenib on the drug response 
and the expression levels of drug resistance-related genes were analyzed using RT-qPCR.. ${ }^{*} \mathrm{P}<0.001$, $* * * P<0.0001$.

a

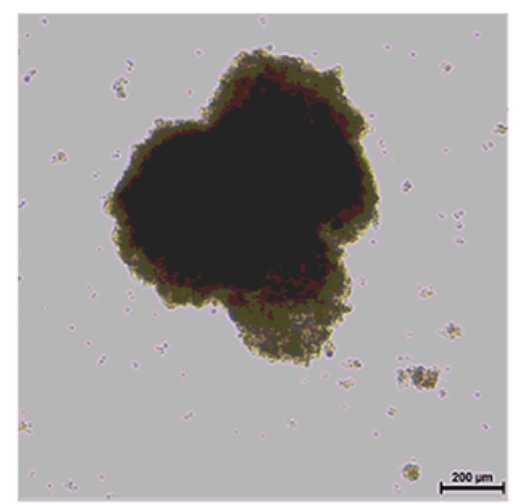

Control-SNU398

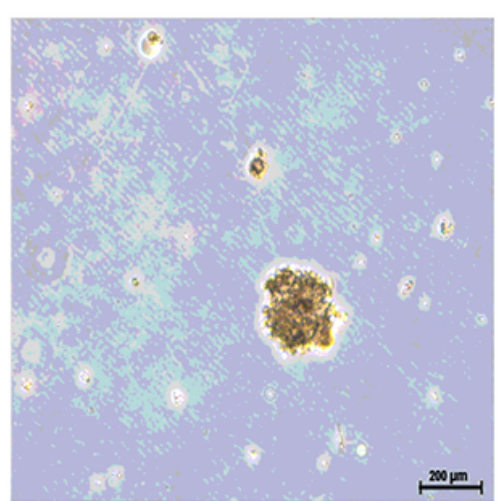

shSIX1-SNU398 b

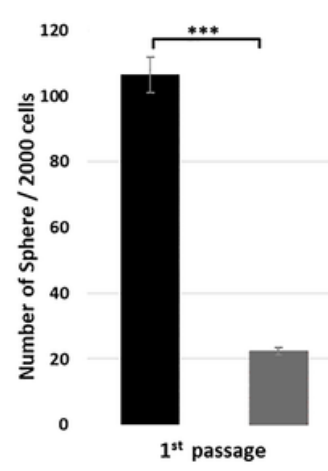

Control-SNU398

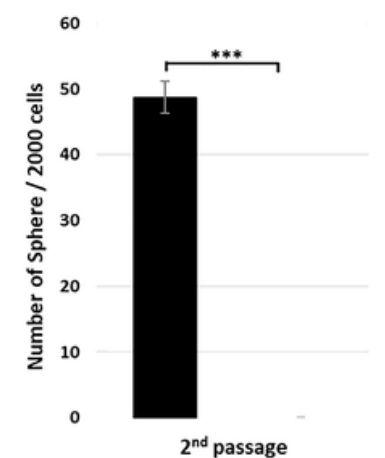

shSIX1-SNU398
C

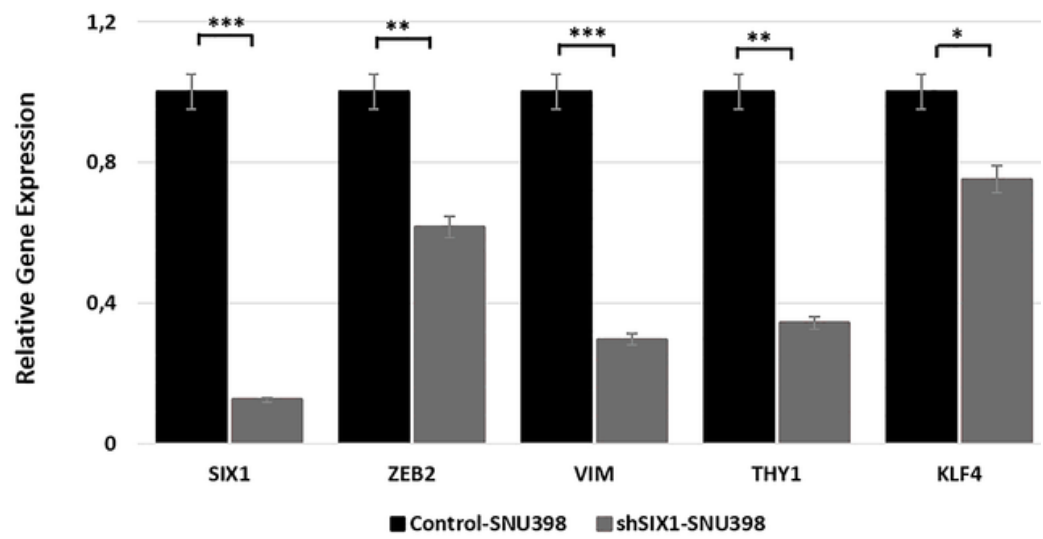

d

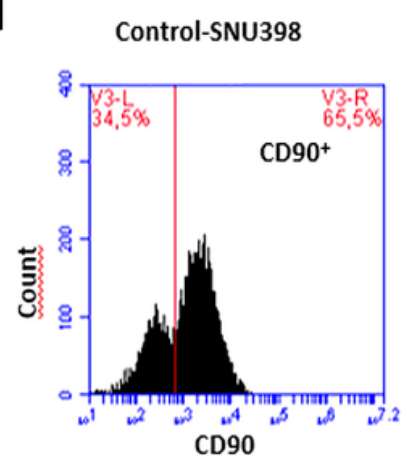

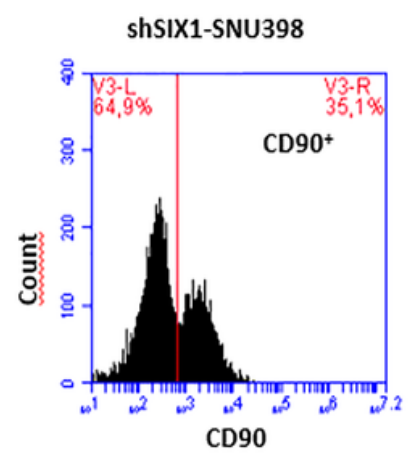

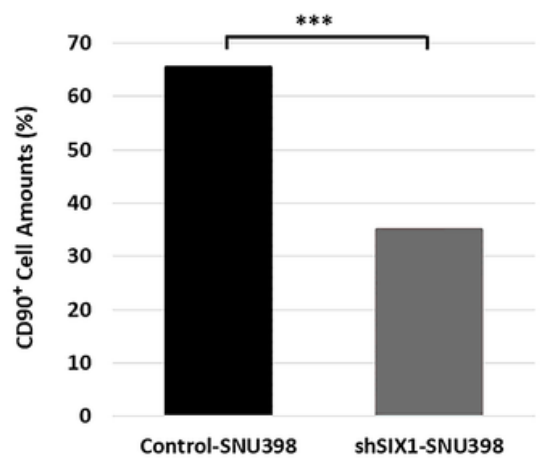

Figure 5

Determining the effect of SIX1 on sphere formation and self-renewal ability. a Sphere formation was measured in shSIX1-SNU398 and control-SNU398 cells. The sphere size decreased in the shSIX1-SNU398 cell line. $b$ To determine the effect of SIX1 on the self-renewal capacity following the sphere formation assay, these cells were passaged two times. It was subsequently observed that the shSIX1-SNU398 cells were unable to self-renew. c RT-qPCR was used to analyze the expression levels of SIX1, ZEB2, VIM, KLF4 ,and THY1 genes in sphere-forming cells. The gene expression analysis revealed that the expression levels of SIX1, ZEB2, VIM, KLF4, and THY1 were significantly downregulated in shSIX1-SNU398 sphere- 
forming cells. d CD90 cell population in shSIX1-SNU398 and control-SNU398 cells was analyzed using flow cytometry. The CD90+ subpopulation was decreased in shSIX1-SNU398 cells compared with the control-SNU398 cells. The differences between groups were analyzed using a Student's t-test. ${ }^{*} \mathrm{P}<0.01$, $\star * \star P<0.0001$. Magnification, $x 5$; scale bars, $200-\mu \mathrm{m}$.

\section{Supplementary Files}

This is a list of supplementary files associated with this preprint. Click to download.

- Fig.S1.docx

- TableS1.docx

- Tables2.docx 